\title{
Effects of anesthesia with sevoflurane and propofol on the cytokine/chemokine production at the airway epithelium during esophagectomy
}

\author{
SAIKO WAKABAYASHI ${ }^{1}$, KEISUKE YAMAGUCHI ${ }^{1}$, SEIICHIRO KUMAKURA ${ }^{1}$, TAISUKE MURAKAMI ${ }^{2}$, \\ AKIMASA SOMEYA $^{2}$, YOSHIAKI KAJIYAMA ${ }^{3}$, ISAO NAGAOKA ${ }^{2}$ and EIICHI INADA ${ }^{1}$ \\ Departments of ${ }^{1}$ Anesthesiology and Pain Medicine, ${ }^{2}$ Host Defense and Biochemical Research, ${ }^{3}$ Esophageal and \\ Gastroenterological Surgery, Juntendo University Graduate School of Medicine, Bunkyo-ku, Tokyo 113-8431, Japan
}

Received November 20, 2013; Accepted April 8, 2014

DOI: $10.3892 /$ ijmm.2014.1762

\begin{abstract}
Post-operative pulmonary complications such as pneumonia, acute lung injury (ALI) and acute respiratory distress syndrome (ARDS) are closely associated with morbidity and mortality after esophagectomy. One lung ventilation (OLV) is commonly used during esophagectomy. However, the effect of the anesthetic agents on the inflammatory response induced by OLV has yet to be evaluated, particularly during esophagectomy, which causes several complications in the lung. The aim of the present study was to determine the effects of anesthetic agents, such as sevoflurane or propofol, on the inflammatory reactions at the airway. Twenty patients undergoing esophagectomy were randomized to receive either sevoflurane $(n=10)$ or propofol $(n=10)$ as a main anesthetic agent. Epithelial lining fluid (ELF) was obtained from ventilated-dependent lung (DL) and collapsed non-dependent lung (NDL) by a bronchoscopic microsampling method. The levels of inflammatory cytokines and chemokine [tumor necrosis factor (TNF)- $\alpha$, interleukin (IL)-1 $\beta$, IL-6, IL-8, IL-10 and IL-12p70] in the ELF were measured using multiplexed bead-based immunoassays before and after OLV. The results indicated that the levels of IL-6 in ELF were significantly increased in both the ventilated DL and collapsed NDL after OLV compared with the levels prior to OLV in the sevoflurane group. By contrast, there was no significant change in the IL-6 levels in the propofol group in the ventilated DL and collapsed NDL before and after OLV. Similarly, IL-8 levels were markedly increased in the ventilated DL and collapsed NDL after OLV compared with those before OLV in the sevoflurane group, whereas there was no significant change in IL-8 levels
\end{abstract}

Correspondence to: Dr Isao Nagaoka, Department of Host Defense and Biochemical Research, Juntendo University, Graduate School of Medicine, 2-1-1 Hongo, Bunkyo-ku, Tokyo 113-8421, Japan E-mail: nagaokai@juntendo.ac.jp

Key words: sevoflurane, propofol, airway epithelium, cytokine, chemokine in the propofol group in the ventilated DL and collapsed NDL before and after OLV. In contrast to the changes in IL-6 and IL-8 levels, levels of IL-10, an anti-inflammatory cytokine, were not obviously changed in both the ventilated DL and collapsed NDL before and after OLV in the sevoflurane group. However, IL-10 levels in the propofol group were increased in the ventilated DL and collapsed NDL after OLV compared with those before OLV. Of note, the levels of TNF- $\alpha$, IL-1 $\beta$ and IL-12p70 in ELF were below the detection limits. These observations suggested that propofol anesthesia more potently suppresses the surgical stress-induced inflammatory perturbation at the local milieu of the airway during esophagectomy compared with sevoflurane anesthesia.

\section{Introduction}

Esophagectomy is one of the most invasive treatments in gastrointestinal surgery (1). Post-operative pulmonary complications such as pneumonia, acute lung injury (ALI) and acute respiratory distress syndrome (ARDS) prolong the duration of mechanical ventilation and have been found to be strongly associated with increased morbidity and mortality after esophagectomy (2). Surgical procedures for esophagectomy also induce systemic inflammatory response syndrome (SIRS), characterized by the overproduction of pro-inflammatory cytokines, which elicit excessive stress and may trigger postoperative complications (2).

Previously, we showed that one-lung ventilation (OLV) more potently induced pulmonary inflammation [interleukin (IL)-6 production] in the ventilated-dependent lung (DL) compared with the collapsed non-dependent lung (NDL) during lung resection (3). Moreover, the inflammatory response in the ventilated DL was significantly suppressed by volatile anesthetic agents (such as sevoflurane and desflurane) compared with an intravenous anesthetic agent (such as propofol) in patients undergoing lung resection (4-7). However, the effect of those anesthetic agents on the inflammatory response in the ventilated DL and collapsed NDL induced by OLV has yet to be evaluated, particularly during esophagectomy that causes several complications in the lung. The aim of this study was to compare the actions of anesthetic agents, sevoflurane 
and propofol on inflammatory reaction in the ventilated DL and collapsed NDL during esophagectomy. For this purpose, airway epithelial lining fluid (ELF) was obtained by a bronchoscopic microsampling method before and after esophagectomy anesthetized with sevoflurane or propofol, and the in vivo production of inflammatory cytokines and chemokine were measured using cytometric bead array systems, and compared before and after the operation.

\section{Materials and methods}

Subjects. Twenty subjects [18 males and 2 females, age range 52-78; mean age \pm standard deviation (SD), 68.6 \pm 6.4 years] classified as American Society of Anesthesiologists Physical Status category I-II, undergoing cervico-thoraco-abdominal three-field lymph node dissection through a right thoracotomy were recruited in this study. The study protocol was approved by the local ethics committee (Juntendo University Hospital, Tokyo, Japan) and conducted from December, 2011 to November, 2012 in accordance with the principles of the amended Declaration of Helsinki and Ethical Guidelines for Epidemiological Research (http://aje.oxfordjournals.org/ content/170/11/1451.full). Subjects provided written informed consent prior to participating in the study. None of the subjects received premedication.

Exclusion criteria were neurologic or psychiatric disease, cardiac disease classified as NYHA classes II-IV (http:// www.abouthf.org/questions_stages.htm), preoperative severe impairment of respiratory function (such as a vital capacity of $<50 \%$ or a forced expiratory volume in $1 \mathrm{sec}$ of $<50 \%$ of that predicted), and pre-existing coagulopathy or thrombocytopenia. Subjects were also excluded if they exhibited systemic or local active infections (either clinically defined or evidenced by elevated C-reactive protein levels, leukocytosis or body temperature of $>38^{\circ} \mathrm{C}$ ).

Subjects were randomly assigned to a sevoflurane $(n=10)$, and a propofol $(n=10)$ group, using a list of random numbers generated by computer software (Microsoft Excel; Microsoft Corp., Redmond, WA, USA).

Study protocols. All the patients underwent general anesthesia combined with epidural anesthesia. Prior to surgery, a thoracic epidural catheter was inserted into the intervertebral space between $\mathrm{T} 7$ and $\mathrm{T} 8$, or $\mathrm{T} 9$ and $\mathrm{T} 10$ for pain management.

Protocol 1 (sevoflurane group) involved the induction of anesthesia with intravenous injection of propofol $(1-2 \mathrm{mg} / \mathrm{kg}$; AstraZeneca, Osaka, Japan), rocuronium $(0.9 \mathrm{mg} / \mathrm{kg}$; MSD K.K., Tokyo, Japan) and remifentanil (0.15-0.3 $\mu \mathrm{g} / \mathrm{kg} / \mathrm{min}$; Janssen Pharmaceutical K.K., Tokyo, Japan). Each subject was then intubated with an endotracheal tube (Rüsch ${ }^{\circledR}$ endotracheal tube; Teleflex Medical Sdn Bhd, Kamunting, Malaysia), and the position of the endotracheal tube was confirmed by a fiber-optic bronchoscopy (Portable Intubation Fiberscope FI-10RBS; Pentax, Tokyo, Japan). After end-tidal $\mathrm{CO}_{2}$ was confirmed, anesthesia was maintained with the inhalation of sevoflurane (0.8-1.5 minimum alveolar concentration; Maruishi Pharmaceutical Co., Ltd., Osaka, Japan) and intravenous infusion of remifentanil $(0.15-0.5 \mu \mathrm{g} / \mathrm{kg} / \mathrm{min})$.

Protocol 2 (propofol group) involved the induction of anesthesia with intravenous infusion of propofol (using a target-controlled infusion technique with a target concentration of $3-4 \mu \mathrm{g} / \mathrm{ml})$, rocuronium $(0.9 \mathrm{mg} / \mathrm{kg})$ and remifentanil $(0.15-0.5 \mu \mathrm{g} / \mathrm{kg} / \mathrm{min})$. Each subject was then intubated with an endotracheal tube, and end-tidal $\mathrm{CO}_{2}$ was confirmed. Anesthesia was maintained with intravenous infusion of propofol (a target concentration of $2-4 \mu \mathrm{g} / \mathrm{ml}$ ) and remifentanil (0.15-0.5 $\mu \mathrm{g} / \mathrm{kg} / \mathrm{min})$.

Following intubation, the patients were placedina left lateral position. The subjects were ventilated by pressure-controlled ventilation with 4-cm $\mathrm{H}_{2} \mathrm{O}$-positive end-expiratory pressure, and peak inspiratory pressure was maintained at $>20 \mathrm{~cm}$ $\mathrm{H}_{2} \mathrm{O}$ with a tidal volume of $7-10 \mathrm{ml} / \mathrm{kg}$, with the fraction of inspired oxygen $\left(\mathrm{FIO}_{2}\right)$ being maintained at 0.4 . The oxygen saturation was adjusted to $>97 \%$, and the respiratory rate was adjusted in order that normocapnia could be maintained (normal arterial carbon dioxide pressure). General anesthesia was maintained using sevoflurane or propofol, with epidural anesthesia using ropivacaine (Maruishi Pharmaceutical Co., Ltd.) and $3 \mathrm{mg}$ morphine hydrate (Daiichi Sankyo Company, Ltd., Tokyo, Japan).

During esophagectomy, OLV was performed using a Coopdech endobronchial blocker tube (Daiken Medical Co., Ltd, Osaka, Japan), and the $\mathrm{FIO}_{2}$ of the left lung (DL) was controlled to maintain $\mathrm{SaO}_{2}>90 \%$. When the peak inspiratory pressure was $>30 \mathrm{~cm} \mathrm{H}_{2} \mathrm{O}$, the position of an endobronchial blocker tube was confirmed using a fiber-optic bronchoscope. Bronchial suction was subsequently performed, and the tidal volume was reduced to $5-7 \mathrm{ml} / \mathrm{kg}$, when required. Following the thoracic esophagectomy and the posterior mediastinal lymph node dissection, the endobronchial blocker tube was removed and the right lung (collapsed NDL) was manually inflated until visible atelectasis was ameliorated, and two-lung ventilation was resumed. Thereafter, the patients were placed in the supine position, and post-operative analgesia was provided with continuous epidural infusion of $0.2 \%$ ropivacaine plus $5 \mathrm{mg}$ morphine/day. A gastric or colonic tube was constructed at laparotomy, and cervical esophagogastrostomy was performed. After confirming that the forced vital capacity was $>10 \mathrm{ml} / \mathrm{kg}$ and the circulation was stable, the endotracheal tube was removed, and then the patients were transferred to the intensive care unit. ALI and ARDS were evaluated throughout the post-surgical period. ALI and ARDS were defined according to the American European Consensus Conference on ARDS criteria (8). Additional criteria included bilateral infiltrations on plain chest radiographs and no clinical evidence of left atrial hypertension. The duration of SIRS after surgery was evaluated according to the definition of the American College of Chest Physicians/Society of Critical Care Medicine. SIRS is characterized by two or more of the following conditions: i) body temperature $>38^{\circ} \mathrm{C}$ or $<36^{\circ} \mathrm{C}$, ii) heart rate $>90$ beats $/ \mathrm{min}$, iii) respiratory rate $>20$ beats/min or $\mathrm{PaCO}_{2}<32 \mathrm{~mm} \mathrm{Hg}$ and iv) white blood cell count $>12,000$ cells $/ \mathrm{mm}^{3},<4,000$ cells $/ \mathrm{mm}^{3}$ or $10 \%$ immature (band) forms (9).

Bronchoscopic microsampling. ELF was obtained by a bronchoscopic microsampling method before and after OLV. A bronchofiberscope was inserted into the trachea through an endotracheal tube and placed at the bifurcation. A bronchoscopic microsampling probe (BC-402C; Olympus, Tokyo, 
Japan) was inserted into the right and left main bronchi through the channel of the bronchofiberscope: the probe comprised a 2.6-mm outer-diameter polyethylene sheath and an inner $1.9-\mathrm{mm}$ diameter cotton probe $(20 \mathrm{~mm}$ length) attached to a stainless steel guidewire. The inner probe was gently advanced $7 \mathrm{~cm}$ from the bifurcation into the right and left main bronchi until it made contact with the mucosal surface. ELF was obtained from the mucosal surface under direct observation. The inner probe was then withdrawn, and the probe was sectioned at $30 \mathrm{~mm}$ from its tip and stored at $-80^{\circ} \mathrm{C}$ until analysis. Furthermore, peripheral blood was collected before and after OLV, simultaneously with ELF sampling, and one day after the surgery. Sera were prepared from blood samples by centrifugation at $1,700 \mathrm{x} \mathrm{g}$ for $20 \mathrm{~min}$ and stored at $-80^{\circ} \mathrm{C}$ until analysis.

Measurement of cytokines. The probe was weighed and mixed with $500 \mu 1$ distilled water by vortexing for $1 \mathrm{~min}$, and the solution was recovered. The probe was then dried and reweighed to estimate the recovered ELF by subtracting the weight of dried probes from that of wet probes (the difference of the weight of wet probe and dried probe was $\sim 10 \mathrm{mg}$ ), after which the dilution factor was calculated (10).

Inflammatory cytokine and chemokine levels in ELF were measured using the cytometric bead array systems (Human Inflammatory Cytokine CBA kit; Becton-Dickinson, Franklin Lakes, NJ, USA). The human Inflammatory Cytokine kit included six fluorescently distinguishable capture microbeads coated with antibodies against the analytes, tumor necrosis factor (TNF)- $\alpha$, IL-1 $\beta$, IL-6, IL-8, IL-10 and IL-12p70. This method can detect cytokines bound onto microbeads by using an enzyme-linked immunosorbent assay. The minimum quantifiable levels of cytokines detected with the Human Inflammatory Cytokine kit were 3.7 for TNF- $\alpha, 7.2$ for IL-1 $\beta$, 2.5 for IL-6, 3.6 for IL-8, 3.3 for IL-10 and $1.9 \mathrm{pg} / \mathrm{ml}$ for IL-12p70. The cytokine levels in sera before and after OLV were also measured using the cytometric bead array systems without dilution.

Statistical analysis. Data are shown as the means \pm SD. Statistical significance was determined by one-way ANOVA, the Student's t-test or the Fisher's exact test (GraphPad Prism 5; GraphPad, San Diego, CA, USA). P $<0.05$ was considered to be significant.

\section{Results}

Patient characteristics. Patient characteristics are shown in Table I. Gender, age, body mass indices, vital capacity, forced expiratory volume in $1 \mathrm{sec}$ (FEV1.0), partial pressure of oxygen in arterial blood $\left(\mathrm{PaO}_{2}\right)$, the durations of surgery, anesthesia and OLV, estimated blood loss and the volume of fluid administration (crystalloid solution and Hespander fluid solution containing hydroxyethyl starch; Otsuka Pharmaceutical Factory, Inc., Tokushima, Japan) and blood transfusion during surgery did not significantly differ between the sevoflurane and propofol groups.

Comparison of cytokine levels in ELF between the sevoflurane and propofol groups before and after OLV. Fig. 1 shows
Table I. Clinical characteristics and surgical data of the sevoflurane and propofol groups.

\begin{tabular}{lcc}
\hline Characteristics & $\begin{array}{c}\text { Sevoflurane } \\
(\mathrm{n}=10)\end{array}$ & $\begin{array}{c}\text { Propofol } \\
(\mathrm{n}=10)\end{array}$ \\
\hline Gender (male/female) & $9 / 1$ & $9 / 1$ \\
Age (years) & $70.7 \pm 5.1$ & $66.4 \pm 7.1$ \\
Body mass index $\left(\mathrm{kg} / \mathrm{m}^{2}\right)$ & $22.0 \pm 2.5$ & $21.7 \pm 2.1$ \\
Vital capacity $(\%)$ & $104 \pm 13$ & $103 \pm 13$ \\
FEV1.0 $(\%)$ & $72 \pm 8$ & $71 \pm 8$ \\
PaO $(\mathrm{mm}$ Hg) & $88 \pm 7$ & $93 \pm 13$ \\
Duration of anesthesia (min) & $485.2 \pm 60.7$ & $529.6 \pm 78.4$ \\
Duration of surgery (min) & $389.5 \pm 59.6$ & $422.7 \pm 35.4$ \\
Duration of OLV (min) & $190.4 \pm 39.6$ & $191.2 \pm 39.1$ \\
Blood loss (ml) & $777 \pm 582$ & $1052 \pm 889$ \\
Fluid administration (ml) & $4432 \pm 660$ & $5607 \pm 1690$ \\
Blood transfusion (ml) & $106 \pm 257$ & $144 \pm 455$ \\
\hline
\end{tabular}

Data are expressed as the means \pm SD. FEV1.0, forced expiratory volume in $1 \mathrm{sec} . \mathrm{PaO}_{2}$, partial pressure of oxygen in arterial blood; $\mathrm{OLV}$, one-lung ventilation.

the changes in the levels of IL-6 in ELF recovered before and after OLV from the ventilated DL and collapsed NDL of the sevoflurane and propofol groups. The levels of IL-6 in ELF were significantly increased in the ventilated DL and collapsed NDL after OLV compared with levels prior to OLV in the sevoflurane group $(\mathrm{P}<0.05)$ (Fig. 1A and $\mathrm{B})$. In contrast, there was no significant change in the IL-6 levels in the propofol group in both the ventilated DL and collapsed NDL before and after OLV (Fig. 1C and D), although the IL-6 level was slightly decreased in the ventilated DL after OLV (Fig. 1C).

Fig. 2 shows the changes in the levels of IL-8 in ELF recovered before and after OLV from the ventilated DL and collapsed NDL of the sevoflurane and propofol groups. Similar to the changes in IL-6 levels, the IL-8 levels were markedly increased in the ventilated DL and collapsed NDL after OLV compared with those prior to OLV in the sevoflurane group (Fig. 2A and B), and the increase in the IL-8 level after OLV was significant in the ventilated DL $(\mathrm{P}<0.05)$ (Fig. 2A). By contrast, there was essentially no significant change in the IL-8 levels in the propofol group in the ventilated DL and collapsed NDL before and after OLV (Fig. 2C and D), although the IL-8 levels were slightly decreased in the ventilated DL and collapsed NDL after the OLV.

Fig. 3 shows the changes in the levels of IL-10 in ELF recovered before and after OLV from the ventilated DL and collapsed NDL of the sevoflurane and propofol groups. In contrast to the changes in IL-6 and IL- 8 levels, no clear change was observed in the IL-10 levels in the ventilated DL and collapsed NDL before and after OLV in the sevoflurane group (Fig. 3A and B). IL-10 levels in the propofol group, however, were increased in the ventilated DL and collapsed NDL after OLV compared with those prior to OLV (Fig. 3C and D), although the changes were not significant. The levels 

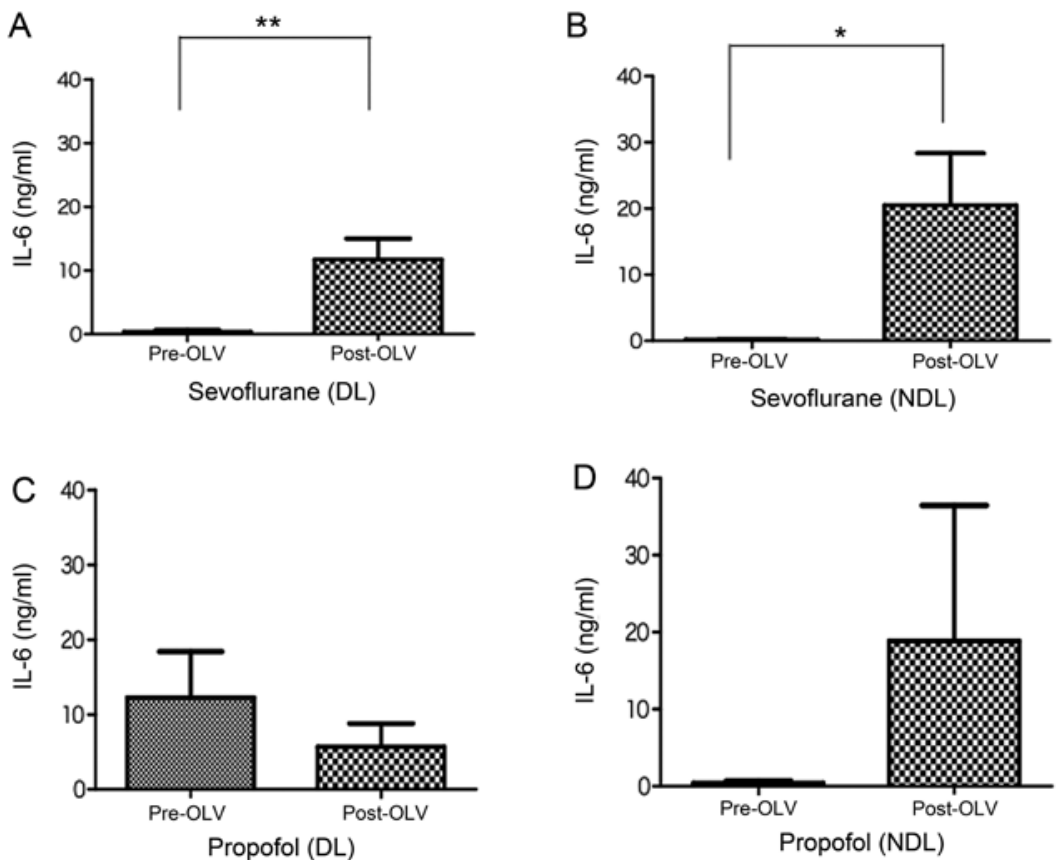

Figure 1. The levels of interleukin (IL)-6 in the epithelial lining fluid (ELF) prior to and following sevoflurane or propofol anesthesia. The levels of IL-6 were measured with a CBA system using ELF recovered from the ventilated-dependent lung (DL) (A and C) and collapsed non-dependent lung (NDL) (B and D) of patients undergoing esophagectomy prior to [pre-one-lung ventilation (OLV)] and following (post-OLV) sevoflurane anesthesia (A and B) or propofol anesthesia $(\mathrm{C}$ and $\mathrm{D})$. Data are expressed as the means \pm standard deviation $(\mathrm{SD})$ and compared between the pre- and post-OLV levels. ${ }^{*} \mathrm{P}<0.05,{ }^{* * *} \mathrm{P}<0.01$.
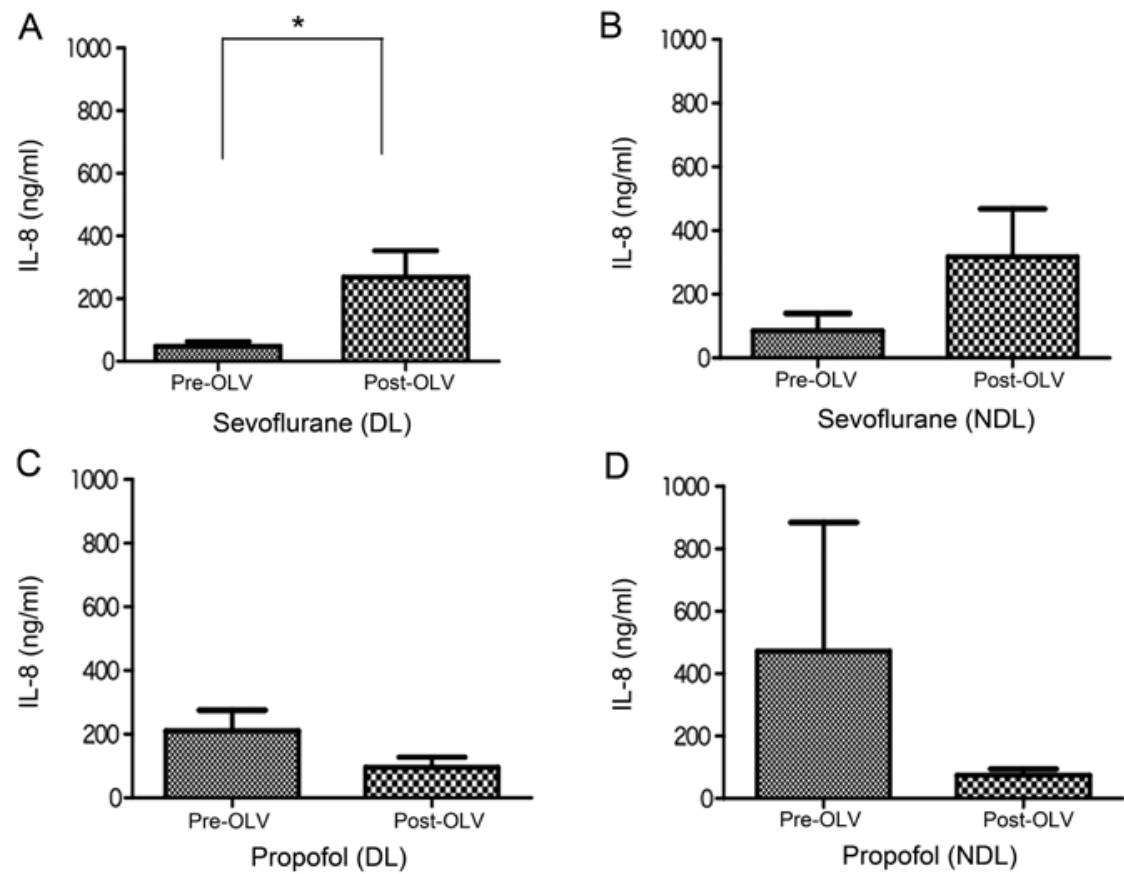

Figure 2. The levels of interleukin (IL)-8 in the epithelial lining fluid (ELF) prior to and following sevoflurane or propofol anesthesia. The levels of IL-8 were measured with a CBA system using ELF recovered from the ventilated dependent lung (DL) (A and C) and collapsed non-dependent lung (NDL) (B and D) of patients undergoing esophagectomy prior to [pre-one-lung ventilation (OLV)] and following (post-OLV) sevoflurane anesthesia (A and B) or propofol anesthesia $(\mathrm{C}$ and $\mathrm{D})$. Data are expressed as the means \pm standard deviation $(\mathrm{SD})$ and compared between the pre- and post-OLV levels. ${ }^{*} \mathrm{P}<0.05$.

of TNF- $\alpha$, IL-1 $\beta$ and IL-12p70 in ELF were below the detection limits.

Comparison of cytokine levels in sera between the sevoflurane and propofol groups before and after OLV. Fig. 4 shows the changes in the serum levels of IL- 6 and IL- 8 before and after
OLV, and one day after the surgery in the sevoflurane and propofol groups. In the sevoflurane and propofol groups, the serum levels of IL- 6 and IL- 8 were markedly increased after OLV compared with those prior to OLV, and slightly decreased one day after the surgery (Fig. 4A and B). Notably, the serum levels of IL- 6 and IL- 8 before and after OLV and one day 

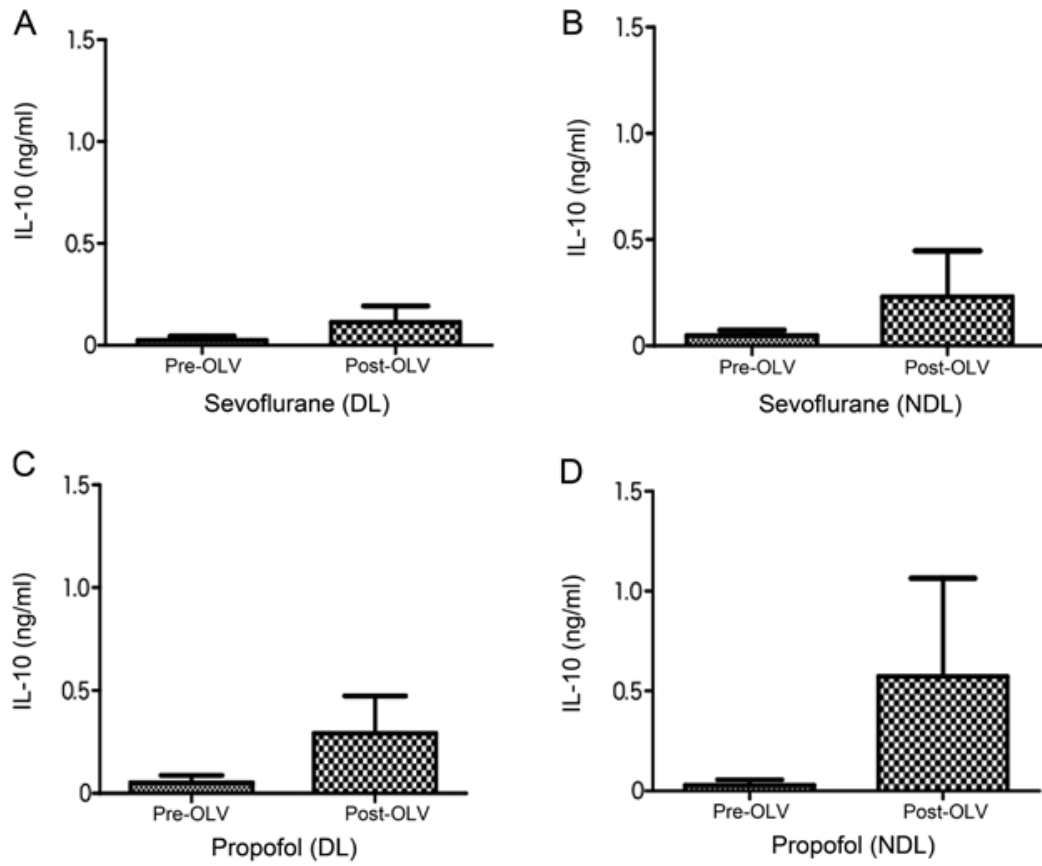

Figure 3. The levels of interleukin (IL)-10 in the epithelial lining fluid (ELF) prior to and following sevoflurane or propofol anesthesia. The levels of IL-10 were measured with a CBA system using ELF recovered from the ventilated-dependent lung (DL) (A and C) and collapsed non-dependent lung (NDL) (B and D) of patients undergoing esophagectomy prior to [pre-one-lung ventilation (OLV)] and following (post-OLV) sevoflurane anesthesia (A and B) or propofol anesthesia (C and D). Data are expressed as the means \pm standard deviation (SD) and compared between the pre- and post-OLV levels.

Table II. Development of SIRS during the post-operative course in sevoflurane and propofol groups.

\begin{tabular}{lccc}
\hline Groups & Post-OLV & POD1 & POD2 \\
\hline Sevoflurane & 2 & 2 & 3 \\
Propofol & 1 & 2 & 4 \\
\hline
\end{tabular}

Number of patients who developed systemic inflammatory response syndrome (SIRS) following one-lung ventilation (OLV) (Post-OLV), one day (POD1) and two days after the surgery (POD2) is shown. Values are compared between the sevoflurane and propofol groups.

after the surgery were not significantly different between the sevoflurane and propofol groups. The levels of TNF- $\alpha$, IL-1 $\beta$, IL-10 and IL-12p70 in sera were below the detection limits.

Changes in the $\mathrm{PaO} 2 / \mathrm{FiO} 2$ ratio during and after the surgery. Fig. 5A shows the changes in the ratio of $\mathrm{PaO}_{2} / \mathrm{FiO}_{2}$, one of the indicators for ALI and ARDS, before and after OLV, one and two days after the surgery in the sevoflurane and propofol groups. The $\mathrm{PaO}_{2} / \mathrm{FiO}_{2}$ ratio was significantly decreased after OLV compared with those before OLV $(\mathrm{P}<0.01)$, and maintained at almost the same level one and two days after the surgery in the sevoflurane and propofol groups. The $\mathrm{PaO}_{2} / \mathrm{FiO}_{2}$ ratio was not significantly different between the sevoflurane and propofol groups at each point (Fig. 5).

Durations of ALI, ARDS and SIRS after the surgery. The durations of ALI, ARDS and SIRS were not statistically different between the sevoflurane and propofol groups after the surgery (Fig. 6). In addition, there were a few incidences of SIRS in the two groups (Table II), although there was no significant difference between the two groups.

\section{Discussion}

Findings of previous studies have shown that airway epithelial cells express and secrete various inflammatory and immune molecules including cytokines (TNF- $\alpha$, IL-1 $\beta$, IL-6 and IL-10) and chemokines (IL- 8 and MCP-1) $(5,11,12)$. TNF- $\alpha$, IL-1 $\beta$ and IL-6 function as proinflammatory molecules, whereas IL-8 and MCP-1 act as chemoattractants that are responsible for the recruitment of effector cells such as neutrophils and monocytes $(13,14)$. Moreover, IL-10 suppresses the proinflammatory cytokine production and the antigen-presenting capacity of monocytes/macrophages and dendritic cells (15). The expression and production of these molecules signifies that the airway epithelium plays an important role in the initiation and exacerbation of inflammatory responses within the airways (16).

Anesthesia and surgical trauma induce immunological and inflammatory responses by stimulating airway epithelial cells to produce pro- and anti-inflammatory cytokines in the lung (17). In this context, anesthetic agents such as sevoflurane and propofol modulate the inflammatory reactions in the local milieu of the airway (4). Moreover, esophagectomy is one of the most invasive treatments in gastrointestinal surgery (1), triggering the inflammatory reaction, with the increased levels of pro-inflammatory cytokines IL-6 and IL-8, and an anti-inflammatory cytokine IL-10 in bronchoalveolar lavage $(18,19)$. In addition, OLV, an anesthetic procedure, induces the inflammatory reaction more potently in the ventilated DL compared with the collapsed NDL (3). Thus, in the 

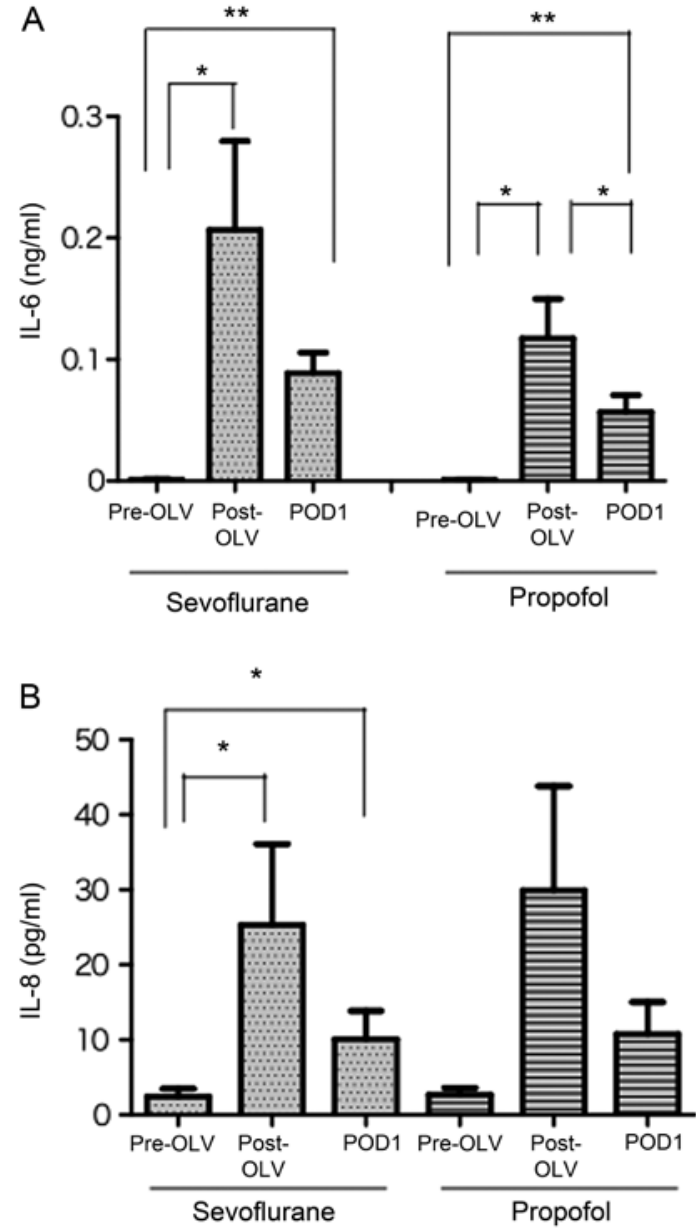

Figure 4. The levels of interleukin (IL)-6 and IL-8 in sera prior to and following sevoflurane or propofol anesthesia. The levels of (A) IL-6 and (B) IL- 8 were measured with a CBA system using sera of patients undergoing esophagectomy prior to [pre-one-lung ventilation (OLV)] and following OLV (post-OLV), one day after the surgery (POD1) in the sevoflurane and propofol groups. Data are expressed as the means \pm standard deviation (SD) and compared among Pre-OLV, Post-OLV and POD1. ${ }^{*} \mathrm{P}<0.05,{ }^{* *} \mathrm{P}<0.01$.

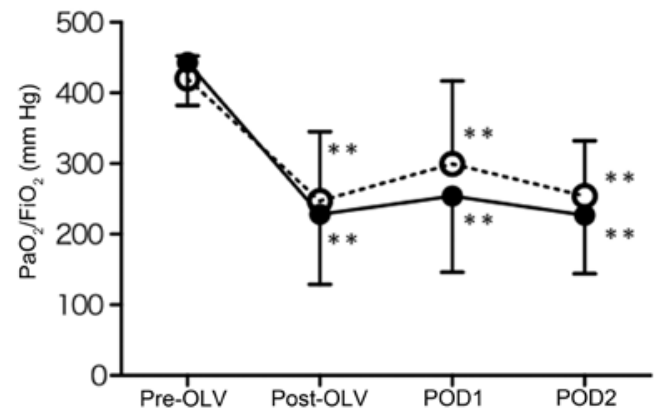

Figure 5. Change in the levels of the partial pressure of oxygen in arterial blood $\left(\mathrm{PaO}_{2}\right)$ /inspired oxygen $\left(\mathrm{FIO}_{2}\right)$ ratio prior to and following sevoflurane or propofol anesthesia. Change in the levels of the $\mathrm{PaO}_{2} / \mathrm{FiO}_{2}$ ratio prior to [pre-one-lung ventilation (OLV)] and following OLV (post-OLV), one day (POD1) and two days after the surgery (POD2) in the sevoflurane (open circles) and propofol groups (closed circles) is shown. Data are expressed as the means \pm standard deviation (SD) and compared between Pre-OLV and Post-OLV, POD1 or POD2. ${ }^{* *} \mathrm{P}<0.01$.

present study, we compared the actions of anesthetic agents, sevoflurane and propofol on the inflammatory reaction in
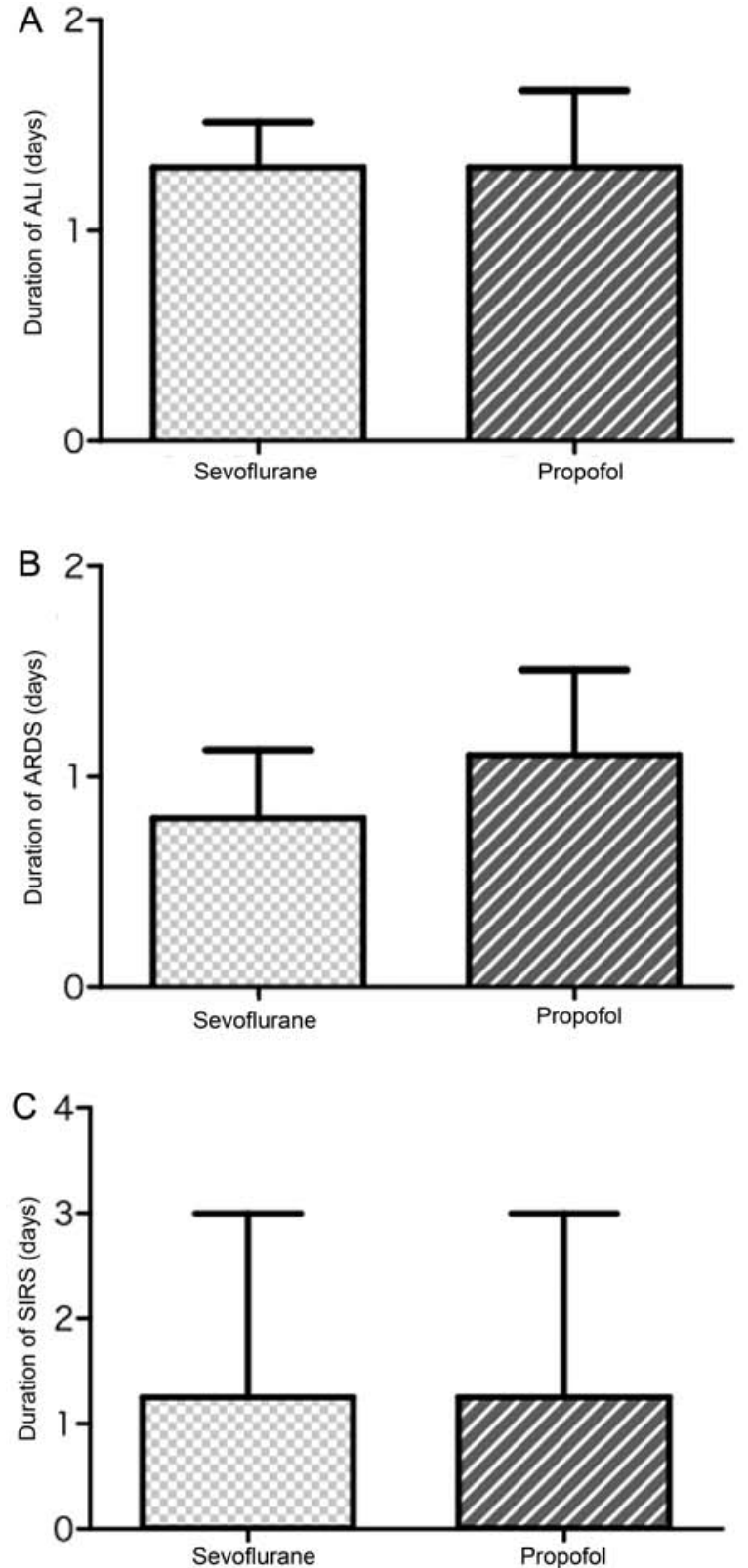

Figure 6. Duration of acute lung injury (ALI), acute respiratory distress syndrome (ARDS) and systemic inflammatory response syndrome (SIRS) in the sevoflurane and propofol groups. The duration of (A) ALI, (B) ARDS and (C) SIRS is shown. Data are expressed as means \pm standard deviation (SD), and compared between the sevoflurane and propofol groups.

the ventilated DL and collapsed NDL during esophagectomy before and after OLV.

The present results show that the levels of IL-6 in ELF were significantly increased in the ventilated DL and collapsed NDL after OLV compared with those prior to OLV in the sevoflurane group. By contrast, no significant change was observed in the IL-6 levels in the propofol group in the ventilated DL and collapsed NDL before and after OLV. Similar to the changes in IL-6 levels, the IL-8 levels were markedly increased in the ventilated DL and collapsed NDL after OLV compared with those prior to OLV in the sevoflurane group, whereas there was no significant change in the IL-8 levels in the propofol group in the ventilated DL and collapsed NDL before and after OLV. In contrast 
to the changes in IL-6 and IL-8 levels, no clear change was evident in the IL-10 levels in the ventilated DL and collapsed NDL before and after OLV in the sevoflurane group. IL-10 levels in the propofol group, however, were increased in the ventilated DL and collapsed NDL after OLV compared with those before OLV. These observations suggest that propofol anesthesia more potently suppresses surgical stress-induced inflammatory perturbation compared with sevoflurane anesthesia during esophagectomy. In this context, it is of note that propofol decreases the secretion of IL-8 from lipopolysaccharide (LPS)-stimulated human neutrophils $(20,21)$. It is reported that propofol, at clinically relevant concentrations, can reduce inflammatory responses in LPS-induced alveolar type II cell injury through the downregulation of CD14 and Toll-like receptor 4 expression (22). Furthermore, propofol is indicated to protect endothelial cells against toxic-free radicals in vitro $(23,24)$. By contrast, it has been shown that anesthesia with sevoflurane leads to the depressed bronchociliary clearance compared with propofol in vivo (25), a depression of the bronchial cilia function is associated with the increased rate of pulmonary complications. Sevoflurane anesthesia is reported to increase the plasma levels of IL-6, TNF- $\alpha$ and IL-1 $\beta$ (26-28). In addition to the effects on the inflammatory responses (cytokine/chemokine production), the inhalation anesthetics such as sevoflurane and desflurane, which comes directly into contact with airway epithelium and alveolar macrophages, potently induce apoptosis and result in the decrease of alveolar macrophages in blonchoalveolar fluid (BALF) (29). Propofol, however, which is intravenously administered, exhibits anti-inflammatory and anti-oxidant actions during mechanical ventilation, thus preventing apoptosis of alveolar cells $(30,31)$. Based on these findings, it can be hypothesized that propofol exerts a more protective effect on the local milieu of the airway compared with sevoflurane during surgical stress with esopagectomy.

Damage of the alveolocapillary unit leads to an increase in alveolar permeability and recruitment of neutrophils and monocytes/macrophages into the alveolar space $(32,33)$. During these processes, pro-inflammatory cytokines are released from pulmonary epithelial cells and inflammatory cells, resulting in an excessive inflammatory response such as ARDS (34). By contrast, among cytokines, IL-10 has anti-inflammatory abilities and inhibits the synthesis of pro-inflammatory cytokines (31). Results of a previous study showed an increased anti-inflammatory response with higher levels of IL-10 in patients receiving propofol anesthesia compared with inhalation anesthesia with isoflurane in abdominal surgery (32). In addition, evidence suggests that the anti-inflammatory response with the production of IL-10 is an important factor for reducing the complications of major abdominal surgery, since the lower IL-10/TNF- $\alpha$ ratio increases the occurrence of post-operative complications (33). Similarly, the present study results revealed that the IL-10 levels in the ELF were increased in the ventilated DL and collapsed NDL in the propofol group after OLV compared with the sevoflurane group (Fig. 3C and D). Moreover, IL-6 and IL-8 levels were not essentially increased in the propofol group following OLV compared with the sevoflurane group. These observations suggest that propofol likely reduces the post-operative complications by exhibiting anti-inflammatory action at the airway through the induction of IL-10 production and suppression of IL-6 and IL-8 production, although there was no apparent difference in the clinical course such as durations of ALI, ARDS and SIRDS after the esophagectomy between propofol and sevoflurane anesthesia.

By analyzing the cytokine/chemokine levels in ELF, we revealed that sevoflurane increased IL- 6 and IL- 8 but not IL-10 in ELF after OLV, whereas propofol increased IL-10 but not IL- 6 and IL-8 in ELF after OLV. By contrast, the levels of IL6 and IL- 8 in sera were almost the same between the sevoflurane and propofol groups before and after OLV, IL6 and IL- 8 similarly increased after OLV, and decreased one day after the surgery in the sevoflurane and propofol groups. These observations suggest that the inflammatory response (the cytokine/chemokine production) at the airway cannot be detected by the changes in the cytokine/chemokine levels in sera. Of note, it has been reported that pro-inflammatory cytokines such as IL-1 $\beta$, IL-4, IL-6, TNF- $\alpha$ and IFN- $\gamma$ are not significantly increased in patients undergoing laparoscopic cholecystectomy compared with open cholecystectomy (35). Moreover, the changes of serum cytokoine/chemokine levels indicate the systemic inflammatory response and depend on the invasive surgical procedure $(36,37)$ such as esophagectomy. Thus, the present study suggests that the cytokine/ chemokine levels in ELF may reflect the inflammatory response at the local milieu of the airway more sensitively compared with those in sera during the invasive esophageal surgery.

Previously, it has been shown that OLV more potently induces the IL- 6 production in the ventilated DL compared with the collapsed NDL during lung resection with propofol anesthesia (3), and that the inflammatory response (cytokine production) in DL is significantly suppressed by sevoflurane compared with propofol in patients undergoing lung resection. By contrast, the present study revealed that there was no essential difference in the IL-6 level in ELF between DL and NDL during propofol anesthesia (Fig. 1C and D), and that the cytokine production in DL was suppressed by propofol but not sevoflurane in patients undergoing esophagectomy (Figs. 1, and 2A and C). These discrepancies remain to be elucidated. However, serum cytokine levels were not significantly changed during lung resection $(3,4)$, but were markedly altered during esophagectomy (Fig. 4). These observations likely suggest that esophagectomy with an invasive surgical procedure (including, not only thoracotomy, but also laparotomy) induces a more potent inflammatory response in the body, and modulates the effects of anesthetic agents (sevoflurane and propofol) on the inflammatory response at the airway, differently from lung resection.

In conclusion, we have demonstrated that the administration of propofol suppresses the IL- 6 and IL- 8 production but enhances the IL-10 production in ELF compared with sevoflurane during esophagectomy. Thus, propofol anesthesia may more potently suppress the surgical stress-induced inflammatory perturbation at the local milieu of the airway compared with sevoflurane anesthesia during esophagectomy. However, it is crucial that the role of these anesthetic agents be given more attention in the clinic due to their ability to induce an inflammatory response and possibly affect post-operative pulmonary and systemic complications. 


\section{References}

1. Yamaguchi K, Sugasawa Y, Takeuchi K, et al: Effects of sivelestat on bronchial inflammatory responses after esophagectomy. Int J Mol Med 28: 187-192, 2011.

2. Sato N, Endo S, Kimura Y, et al: Influence of a human protease inhibitor on surgical stress induced immunosuppression. Dig Surg 19: 300-305, 2002.

3. Sugasawa Y, Yamaguchi K, Kumakura S, et al: The effect of one-lung ventilation upon pulmonary inflammatory responses during lung resection. J Anesth 25: 170-177, 2011.

4. Sugasawa Y, Yamaguchi K, Kumakura S, et al: Effects of sevoflurane and propofol on pulmonary inflammatory responses during lung resection. J Anesth 26: 62-69, 2012.

5. De Conno E, Steurer MP, Wittlinger M, et al: Anesthetic-induced improvement of the inflammatory response to one-lung ventilation. Anesthesiology 110: 1316-1326, 2009.

6. Schilling T, Kozian A, Kretzschmar M, et al: Effects of propofol and desflurane anaesthesia on the alveolar inflammatory response to one-lung ventilation. Br J Anaesth 99: 368-375, 2007.

7. Schilling T, Kozian A, Senturk M, et al: Effects of volatile and intravenous anesthesia on the alveolar and systemic inflammatory response in thoracic surgical patients. Anesthesiology 115: 65-74, 2011.

8. Bernard GR, Artigas A, Brigham KL, et al: The AmericanEuropean Consensus Conference on ARDS. Definitions, mechanisms, relevant outcomes, and clinical trial coordination. Am J Respir Crit Care Med 149: 818-824, 1994.

9. American College of Chest Physicians/Society of Critical Care Medicine Consensus Conference: definitions for sepsis and organ failure and guidelines for the use of innovative therapies in sepsis. Crit Care Med 20: 864-874, 1992.

10. Nakayama H, Kitayama J, Muto $T$ and Nagawa $H$ : Characterization of intracellular cytokine profile of $\mathrm{CD}^{+} \mathrm{T}$ cells in peripheral blood and tumor-draining lymph nodes of patients with gastrointestinal cancer. Jpn J Clin Oncol 30: 301-305, 2000.

11. Simon RH and Paine R III: Participation of pulmonary alveolar epithelial cells in lung inflammation. J Lab Clin Med 126: 108-118, 1995.

12. Takizawa H: Airway epithelial cells as regulators of airway inflammation (Review). Int J Mol Med 1: 367-378, 1998.

13. Vozzelli MA, Mason SN, Whorton MH and Auten RL Jr: Antimacrophage chemokine treatment prevents neutrophil and macrophage influx in hyperoxia-exposed newborn rat lung. Am J Physiol Lung Cell Mol Physiol 286: L488-L493, 2004.

14. Beck-Schimmer B, Schwendener R, Pasch T, Reyes L, Booy C and Schimmer RC: Alveolar macrophages regulate neutrophi recruitment in endotoxin-induced lung injury. Respir Res 6: 61, 2005.

15. Asadullah K, Sterry W and Volk HD: Interleukin-10 therapy-review of a new approach. Pharmacol Rev 55: 241-269, 2003.

16. Kumakura S, Yamaguchi K, Sugasawa Y, et al: Effects of nitrous oxide on the production of cytokines and chemokines by the airway epithelium during anesthesia with sevoflurane and propofol. Mol Med Rep 8: 1643-1648, 2013.

17. Kotani N, Hashimoto H, Sessler DI, et al: Intraoperative modulation of alveolar macrophage function during isoflurane and propofol anesthesia. Anesthesiology 89: 1125-1132, 1998.

18. Sato N, Koeda K, Kimura Y, et al: Cytokine profile of serum and bronchoalveolar lavage fluids following thoracic esophageal cancer surgery. Eur Surg Res 33: 279-284, 2001.

19. Abe T, Oka M, Tangoku A, et al: Interleukin-6 production in lung tissue after transthoracic esophagectomy. J Am Coll Surg 192: 322-329, 2001.
20. Galley HF, Dubbels AM and Webster NR: The effect of midazolam and propofol on interleukin-8 from human polymorphonuclear leukocytes. Anesth Analg 86: 1289-1293, 1998.

21. O'Donnell NG, McSharry CP, Wilkinson PC and Asbury AJ: Comparison of the inhibitory effect of propofol, thiopentone and midazolam on neutrophil polarization in vitro in the presence or absence of human serum albumin. Br J Anaesth 69: 70-74, 1992.

22. Ma L, Wu X, Chen W and Fujino Y: Propofol has antiinflammatory effects on alveolar type II epithelial cells. Acta Anaesthesiol Scand 54: 362-369, 2010.

23. Murphy PG, Ogilvy AJ and Whiteley SM: The effect of propofol on the neutrophil respiratory burst. Eur J Anaesthesiol 13: 471-473, 1996

24. Mathy-Hartert M, Mouithys-Mickalad A, Kohnen S, DebyDupont G, Lamy M and Hans P: Effects of propofol on endothelial cells subjected to a peroxynitrite donor (SIN-1). Anaesthesia 55: 1066-1071, 2000.

25. Ledowski T, Paech MJ, Patel B and Schug SA: Bronchial mucus transport velocity in patients receiving propofol and remifentanil versus sevoflurane and remifentanil anesthesia. Anesth Analg 102: 1427-1430, 2006.

26. Takala RS, Soukka HR, Salo MS, et al: Pulmonary inflammatory mediators after sevoflurane and thiopentone anaesthesia in pigs. Acta Anaesthesiol Scand 48: 40-45, 2004.

27. Kotani N, Takahashi S, Sessler DI, et al: Volatile anesthetics augment expression of proinflammatory cytokines in rat alveolar macrophages during mechanical ventilation. Anesthesiology 91: 187-197, 1999.

28. Koksal GM, Sayilgan C, Gungor G, et al: Effects of sevoflurane and desflurane on cytokine response during tympanoplasty surgery. Acta Anaesthesiol Scand 49: 835-839, 2005.

29. Kalimeris K, Christodoulaki K, Karakitsos P, et al: Influence of propofol and volatile anaesthetics on the inflammatory response in the ventilated lung. Acta Anaesthesiol Scand 55: 740-748, 2011.

30. Wei H, Liang G, Yang $\mathrm{H}$, et al: The common inhalational anesthetic isoflurane induces apoptosis via activation of inositol 1,4,5-trisphosphate receptors. Anesthesiology 108: 251-260, 2008.

31. Moore KW, de Waal Malefyt R, Coffman RL and O'Garra A: Interleukin-10 and the interleukin-10 receptor. Annu Rev Immunol 19: 683-765, 2001.

32. Gilliland HE, Armstrong MA, Carabine U and McMurray TJ: The choice of anesthetic maintenance technique influences the antiinflammatory cytokine response to abdominal surgery. Anesth Analg 85: 1394-1398, 1997.

33. Dimopoulou I, Armaganidis A, Douka E, et al: Tumour necrosis factor-alpha ( $\mathrm{TNF} \alpha)$ and interleukin-10 are crucial mediators in post-operative systemic inflammatory response and determine the occurrence of complications after major abdominal surgery. Cytokine 37: 55-61, 2007.

34. Hudson LD, Milberg JA, Anardi D and Maunder RJ: Clinical risks for development of the acute respiratory distress syndrome. Am J Respir Crit Care Med 151: 293-301, 1995.

35. Helmy SA, Wahby MA and El-Nawaway M: The effect of anaesthesia and surgery on plasma cytokine production. Anaesthesia 54: 733-738, 1999.

36. Michelet P, D'Journo XB, Roch A, et al: Protective ventilation influences systemic inflammation after esophagectomy: a randomized controlled study. Anesthesiology 105: 911-919, 2006.

37. D'Journo XB, Michelet P, Marin V, et al: An early inflammatory response to oesophagectomy predicts the occurrence of pulmonary complications. Eur J Cardiothorac Surg 37: 1144-1151, 2010. 